\title{
The Research on Energy-saving Technology of the Set Covering Base Sta- tion in Cellular Networks
}

\author{
Yi Tian*
}

Shangluo University, Shangluo, China

\begin{abstract}
The flow distribution of cellular networks fluctuates according to the changes of time and space, and this phenomenon makes the low utilization rate of some base stations. Although the base stations' utilization rate is low, it causes the large energy consumption. The CSO can save the energy of base stations according to the load, but it is influenced by the load and the sequence of base stations. Aiming at energy-saving, this paper proposes a centralized-greedy-add algorithm based on taking the closure of base stations as the limited capacity set covering model. The simulation under UrbanMicro (UMi) shows that the energy-saving effect obtains a great improvement compared to the sequence method based on loads.
\end{abstract}

Keywords: Cellular networks, the closure of base stations, sequence of base stations, set covering, energy saving.

\section{INTRODUCTION}

The cellular network realizes the overall data covering, which is the best way for internet access, and causes the increasing requirement of the cellular network data rate. For this requirement, the operators are trying to use new technologies and increasing the number of the base stations is one of this. However, the increasing of base stations make the cost high and cause the environment problems [1]. The next generation of green communications network, energy efficiency(E2) has become one of the most important performance index. It predicts that the number of cellular networks will rise rapidly, and become the main source of carbon emissions [2]. The overall cellular energy up to $80 \%$ are obtained from the base station. Therefore, people have put forward several methods to reduce the energy consumption of base station, such as reducing the number of base stations by taking the deployment of high efficiency of hardware to use the solar energy resources [3, 4].

The COS is proposed for energy saving by close a certain number of base stations. The reference [5] introduces a kind of heuristic greedy-drop algorithm named Cell-Zooming, which defines that the closing sequence of the base stations are from the low-load base station until the base station to be closed will influence the other base stations working. The reference [6] proposes the improved Cell-Zooming, which is realized by checking all the base stations before closure. The reference [7] describes greedy-add algorithm, it considers all the requirements and minimizes the number of operating base stations. The algorithm opens the base stations according to the load, when each user is assigned to an active base station, the algorithm terminates. There are other areas of the algorithm to be used for CSO technology, such as efficacy algorithm in literature [8] and general algorithm of Literatures [9]. However, the key factor of closure depends on the adjacent base stations' load and closing sequence as well as itself.

In this paper, three kinds of sequence criteria are researched. The simulation results shows that compared with the current closing strategy which is based on load, the sequencing method based on the number of users may close more base stations. Using [6] cell-zooming improved algorithm as the benchmark algorithm, and comparing it with the algorithm introduced in this paper, the results show that when the number of users in each base station is 10 or more, the performance of the proposed algorithm is superior to the literature [6] benchmark algorithm. the contribution of this paper is as follows: 1) take various method of base stations' sequence as research target, and compare the impact of these standards on energy efficiency. 2) The CSO technology is stated as a kind of set covering problem, which can effectively solved by the greedy-add algorithms.

\section{PROBLEM DESCRIPTIONS}

Symbols and their meanings are shown in Table $\mathbf{1}$.

The purpose of CSO is to close base stations as much as possible on the condition of meeting the user's data rate requirement of the base station. And this purpose can be achieved by using a set covering theory.

The set covering is described as $(U, S, C)$, and $U$ is the whole domain of $n$ elements, $S=\left\{S_{1}, S_{2}, \cdots, S_{m}\right\}$ is the set consisting of $\mathrm{m}$ subsets of $U, C=\left\{c_{1}, c_{2}, \cdots, c_{m}\right\}$ is the cost set of each subset $S_{k} \in S$. Set cover is to determine a subset 
Table 1. Symbols and meanings.

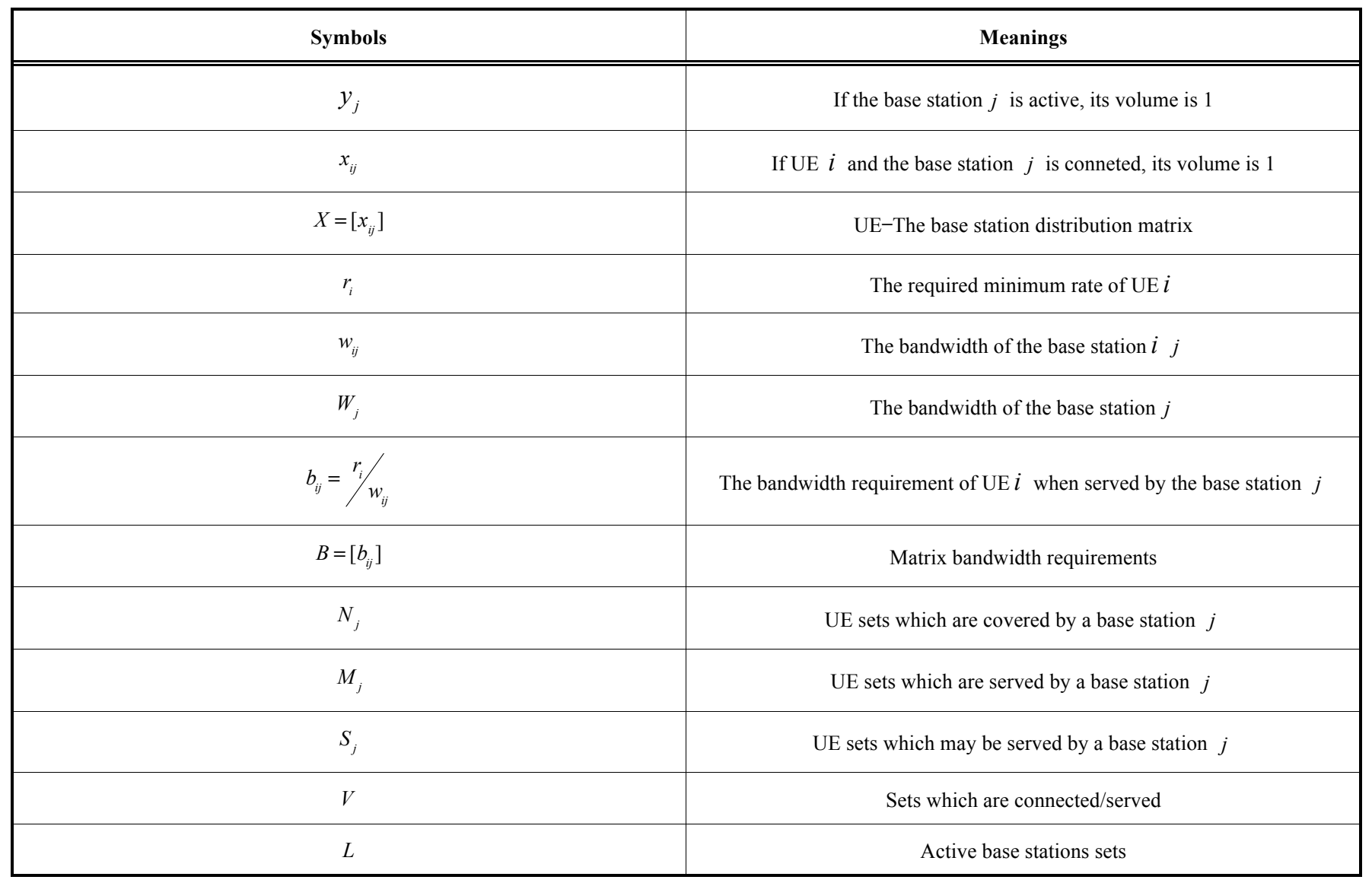

of $S$ that make all elements of $U$ be included in at least one subset. The purpose of set covering problem is to find out a set covering $S^{*} \subseteq S$ to minimize costs, and if all the subsets cost are same, then it becomes the unweighted problem $(U, S, 1)$. The purpose of the unweighted set covering problem is to find a minimum combination of subset $\left\{S_{1}, S_{2}, \cdots, S_{m}\right\}$ which can cover all elements of the $U$.

The set covering of wireless sensor network is in certain extent similar to the CSO technology, and both are based on the purpose of the minimum set covering the same points. However, after the CSO are used as set covering, the steps differ from wireless sensor networks. First, in the CSO problem, the base station capacity is an important constraint, but in a wireless sensor network the data transfer rate is low, so the base station capacity constraint does not exist. Then the point is the model of covering. Coverage area of a wireless sensor network is assumed to be circular area [10], but in the CSO problem due to obstacles, reflection and diffraction and other factors leads to the shadowing effect, so its coverage area is not circular area.

The last point is the requirement of energy-saving. Because of wireless sensor networks' energy scarcity, it must be energy-efficient, while the cellular network is able to carry out energy-saving better.

CSO can be modeled as $(U, S, 1)$ set covering term in (1), and $U$ is the user's equipment set(UEs) in the network, $S$ is the UE subset affiliated to the same base station, and ' 1 ' is unweighted set covering problem

$\min i \operatorname{mize} \sum_{j \in S} y_{j}$

Conditions are: $\sum_{i \in U} b_{i j} x_{i j} \leq W_{j}, \forall j \in S$

$\sum_{j \in U} x_{i j} y_{j} \geq 1, \forall i \in U, \forall j \in S$

$x_{i j}, y_{j} \in\{0,1\}, \forall i \in U, \forall j \in S$

(2) is capacity constraint, it means that subset UE, covered by base station $j$, is contained in the services set $S_{j}$, which avoid the exceeding of the bandwidth $W_{j}$; (3) is the demand ed segmentation probability. In CSO approach, all UE needs to be satisfied by a single base station, which is called the indivisible needs. (4) is the type of demand. For the limited capacity set cover, UE $i$ requires the same requirements $b_{i}$ for all base stations. However, in the cellular network, the UEi's needs is the bandwidth requirement, with different services provided in different base stations, which will be referred as differentiated demand. Thus, notation $b_{i j}$ is used to distinguish different bandwidth requirements for each base station. Although there are a variety of algorithms 
can solve the capacity-constrained set covering problem [11], but these algorithms are targeted, which does not apply to the different needs of the scene.

\section{ALGORITHM IN THIS PAPER}

This paper proposes a dual-stage centralized algorithm. Algorithm 1 set of services in the first stage is responsible for determining implementation of the constraint (2). To determine the set of base stations $j$ 's services $S_{j}$, UE in $M_{j}$ has to be added into $S_{j}$ first, and added new UE to the minimum $b_{i^{*} j}$ requirement $\mathrm{UE} i^{*}$, then fill the base bandwidth $W_{j}$ randomly.

The algorithm stops until the services set $S=\left\{S_{1}, S_{2}, \cdots, S_{m}\right\}$ is gotten, and $\mathrm{m}$ is the number of bases sations.

\begin{tabular}{l} 
Algorithm $1:$ Determining the set of services \\
Input: $B, X, V, W_{j}$ \\
Output: $S=\left\{S_{1}, S_{2}, \cdots, S_{m}\right\}$ services set $\forall j \in S$ \\
$1:$ for each $j$ do \\
$2: S_{j} \leftarrow S_{j} \cup\left\{M_{j} \backslash V\right\}$ \\
$3: Z=\sum_{\forall r \in S_{j}} b_{r^{\prime}}$, the used bandwidth \\
$4:$ while $Z<W_{j}$ do \\
$5:$ looking for $i^{*}=\arg \min _{i^{*} \in\left\{\mathbb{N}_{j} W\right\}} b_{i^{*} j}$ \\
$6:$ if $Z+b_{i^{*} j}<W_{j}$ then \\
$7: Z \leftarrow Z+b_{i^{*} j}$ \\
$8: S_{j} \leftarrow S_{j} \cup\left\{i^{*}\right\}$ \\
$9:$ else \\
$10:$ stop while recyle \\
$11:$ end \\
$12:$ end \\
$13:$ end \\
$14:$ end \\
$15:$ return $S$ \\
\hline
\end{tabular}

In the second stage, the main content is Algorithm 2. Its input is the service set $S$ from Algorithm 1. At the beginning of the algorithm, it is assumed that all base stations are closed, and all the UEs are not connected. At each iteration, the algorithm selects the base station $j^{*}$ to be enabled. The base station's selected sequence (base order) has a significant impact for future base closing decisions. It is common to select one based on its current load. However, the closing station and the base station depends on the load itself and the channel quality, available bandwidth of the neighbor base stations with other base stations between the UE and other factors. Therefore, we study 3 different base station sequencing methods, and analyze their impact on the final number of closing base stations. Base station selecting step of the algorithm, is in 6-16 of Algorithm 2. In addition, three kinds of base sequencing methods are as follows:

(1) Maximum load: In this case, the base station to be selected is the highest load station. It is common to do this in many literatures.

(2) Maximum Users: At this point, select the next station which is receiving its services, and not connected with maximum UE number.

(3) Maximum Center: In this case, the base station selected is the center with maximum number of UE (and good channel quality UE). The guideline emphasizes the optimum choice of channel quality containing the maximum number of UE. If the channel duality of base stations is superior, it means that the channel is not good as other base stations, and therefore it requires relatively more resources to meet requirements. If $w_{i j} \geq w_{t h}$, the center UE of $j$ is UE $i$, and $w_{t h}$ is the spectral efficiency of center UE, which is supposed to be $10 \mathrm{bps} / \mathrm{Hz}$.

After selecting the open base station $j^{*}$, its all service set $\left\{S_{j^{*}}\right\}$ join UE set $V$, and new distribution matrix $X$ is assigned. At each iteration, the service set is updated according to the updated $V$ and $X$ calling Algorithm 1. When all UE connections terminate algorithms, the collection station will remain active, and all other base stations are closed.

\begin{tabular}{|l|}
\hline Algorithm $2:$ Greedy add \\
\hline input $: M_{j}, X, B, \Omega$ \\
$U=\left\{e_{1}, e_{2}, \cdots, e_{n}\right\}$, set of all UE \\
output $: L, X$ \\
$1: L \leftarrow \phi$, active base stations \\
$2: V \leftarrow \phi$, set of base stations connected to \\
$3:$ while $V \neq U$ do \\
$4:$ call Algorithm1, get services set $S$ \\
$5:$ switch sequence method do \\
$6:$ case Maximum load \\
$7:$ look for $j^{*}=\arg \max _{j^{*} \in\{S \backslash L\}}\left(\sum_{\forall i \in\left\{M_{j^{*}} \backslash V\right\}} b_{i j^{*}}\right)$ \\
$8:$ end \\
$9:$ case Maximum $\operatorname{Users}_{j^{*} \in\{S \backslash L\}}\left|S_{j^{*}}\right|$ \\
$10:$ look for $j^{*}={\arg \max }$ \\
\hline
\end{tabular}




$$
\begin{aligned}
& 11: \text { end } \\
& 12: \text { case Maximum Center } \\
& 13: T_{j}=\left\{a \in\left\{M_{j} \backslash V\right\} \mid w_{a j} \geq w_{t h}\right\}, \forall_{j} \in S \\
& 14: \text { look for } j^{*}=\arg \max _{j^{*} \in\{\mathrm{S} \backslash \mathrm{L}\}}\left|T_{j^{*}}\right| \\
& 15: \text { end } \\
& 16: \text { endsw } \\
& 17: L \leftarrow L \cup\left\{j^{*}\right\} \\
& 18: \quad V \leftarrow V \cup\left\{S_{j^{*}}\right\} \\
& 19: \text { update } X \\
& 20: \text { end } \\
& 21: \text { return } L, X
\end{aligned}
$$

\section{EXPERIMENT SIMULATIONS}

Analyze downlink of cellular network containing the omni-directional antenna pattern, which is with 100 base stations. Experiment is done according to the literature [12] assessment criteria, which is through simulating small base (UMi) stations to represent a small base station environment. Due to the presence of buildings in UMi scene, the next line of sight (LOS) signal is not the common path of this environment. The path loss is calculated based on probability of two path loss model and various models: LOS model and non-LOS model. The frequency reuse is 1 , which means each base station uses the entire spectrum. Table 2 shows the simulation necessary parameters. The distance between the stations (stations scale) and transmission power value are corresponding to a small base station. And the requirements of each base station's rate and UE number are set for lowing network load, and this is a typical scenario CSO technology.

We assume that in the simulation covering set of each base station includes all areas of the UE. Because the base station deployment is dense under UMi scenarios, this is a reasonable assumption. In order to reduce the difficulty of the problem, we assume that the interference between base stations (ICI) is controlled through interference management techniques. Considering the ICI, the expansion algorithm is also very simple, the results of the algorithm is essentially very similar to Fig. (1 and 2). Therefore, the spectral efficiency $\left(w_{i j}\right)$ is calculated according to the $S N R_{i j}$ between the $\mathrm{UE}^{i}$ and the base station $j$ with (4).

$w_{i j}=\log _{2}\left(1+S N R_{i j}\right)$

This algorithm belongs to centralized algorithm, then all base stations and a central entity (cloud) are connected, and

\begin{tabular}{|c|c|}
\hline Cellular networks distribution & Box \\
\hline Distance between base stations & $200 \mathrm{~m}$ \\
\hline Antenna Pattern & omnidirectional \\
\hline emission power & $41 \mathrm{dBm}$ \\
\hline Bandwidth & $10 \mathrm{MHz}$ \\
\hline carrier frequency $\left(f_{c}\right)$ & $2.5 \mathrm{GHz}$ \\
\hline UE distribution & stochastic Uniform \\
\hline Probability of interior UE & $50 \%$ \\
\hline User number of each base station & $5,10,15,20,25$ \\
\hline Rate request $r_{i}$ & $500 \mathrm{kpbs}$ \\
\hline UEnoise coefficient & $5 \mathrm{~dB}$ \\
\hline Noise figure & $-174 \mathrm{dBm} / \mathrm{Hz}$ \\
\hline Shadowing effect standard deviation of LOS & $4 \mathrm{~dB}$ \\
\hline Shadowing effect standard deviation of NLOS & $6 \mathrm{~dB}$ \\
\hline Flow pattern & Total queue \\
\hline
\end{tabular}
the entity contains all global information of all UE and all base stations' SINR. Simulation runs 100 times. In each run, $\mathrm{UE}$ in region is random discarded. The average number of the base station is closed to take the mean of the simulation 100 times. Energy saving effect and the number of the closed base station is linearly proportional relationship, because there is no data transmission will consume large amounts of energy, the energy used to transmit data may be ignored. In the deployment of base stations 100 , energy ratio is equal to

Table 2. Simulation parameters. 


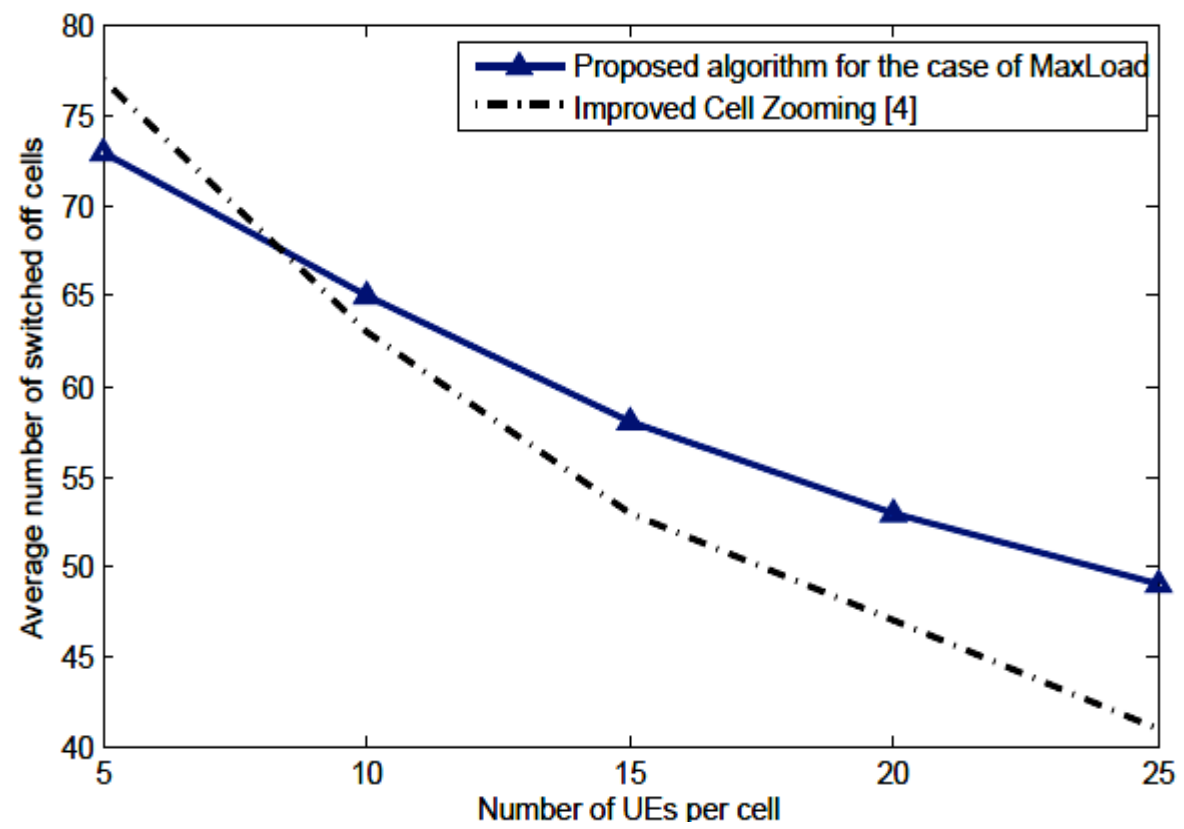

Fig. (1). Energy performance of maximum load sequencing criteria and algorithm [6] baseline algorithm.

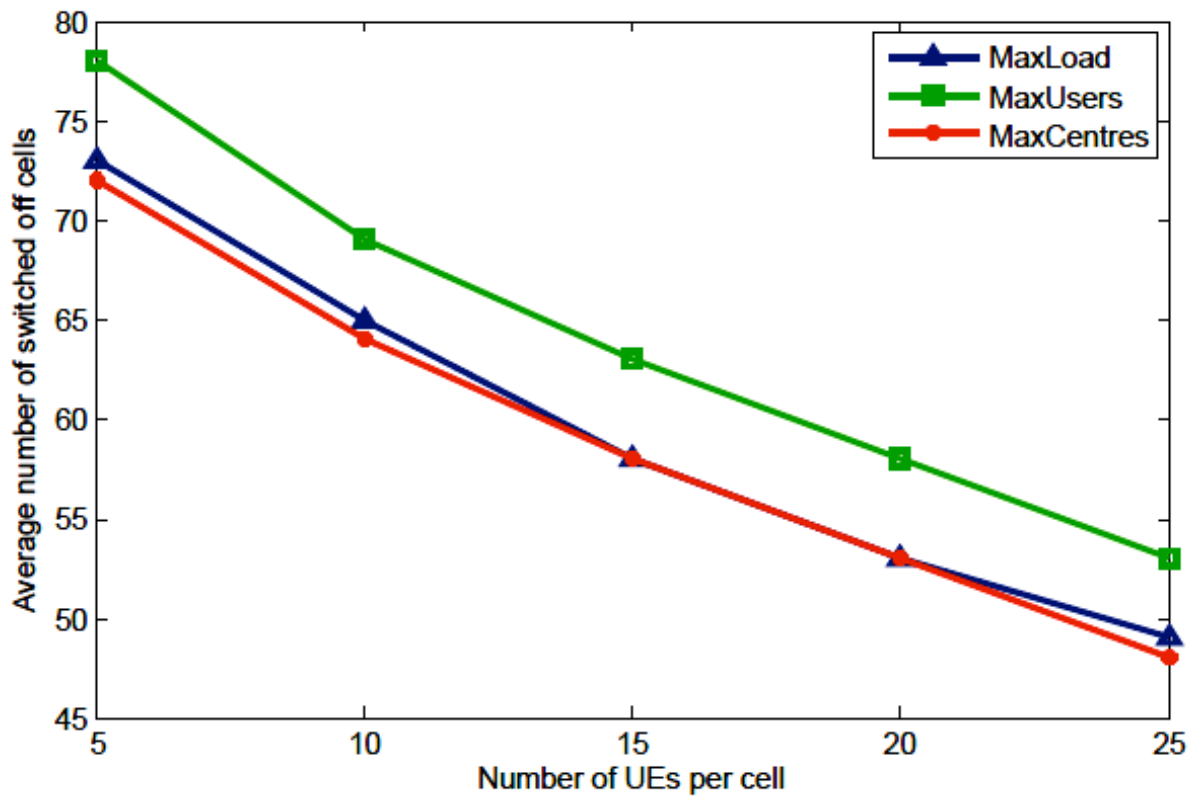

Fig. (2). Energy-saving effect of base stations on different sequencing criteria.

the closed base station number, it means if 30 base stations are closed, the corresponding energy saving effect is $30 \%$.

The comparing the results of the proposed algorithm and the literature [6] greedy-drop baseline algorithm is shown in Fig. (1). The X-axis represents the UE number of each base station, Y-axis represents the energy-saving effect. For fair comparison, we use the same maximum load sequencing criteria according to the load to run the two algorithms. When the UE number of each base station is small, such as only five UE, the performance is slightly better than the benchmark algorithm. However, when the number of each base station UE goes to 10 or more, the performance of the proposed algorithm is superior to the benchmark algorithm, when the UE number of base stations is 25 , this algorithm is higher $20 \%$ more energy savings. The reason to achieve such performance is that the greedy-add strategy with respect to the greedy-drop obtains a performance advantage. For the greedy-drop method, before the station is closed, its UE needs to be handed over. This may cause that the bandwidth application rate of the other base station can not be $100 \%$. Therefore, the focus at this time is trying to remove the base station's load rather than to maximize the load section in the base station. On the other hand, before the station opens, the 


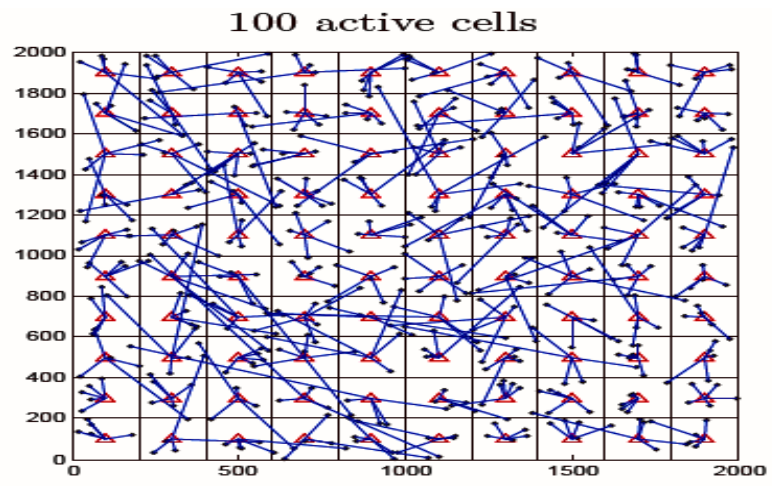

(a) Initial UE- base station distribution

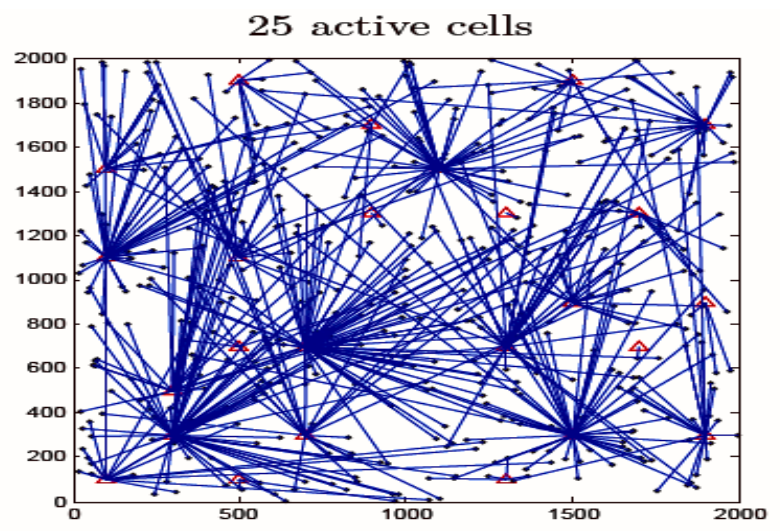

(b) UE based on maximum load- base station distribution

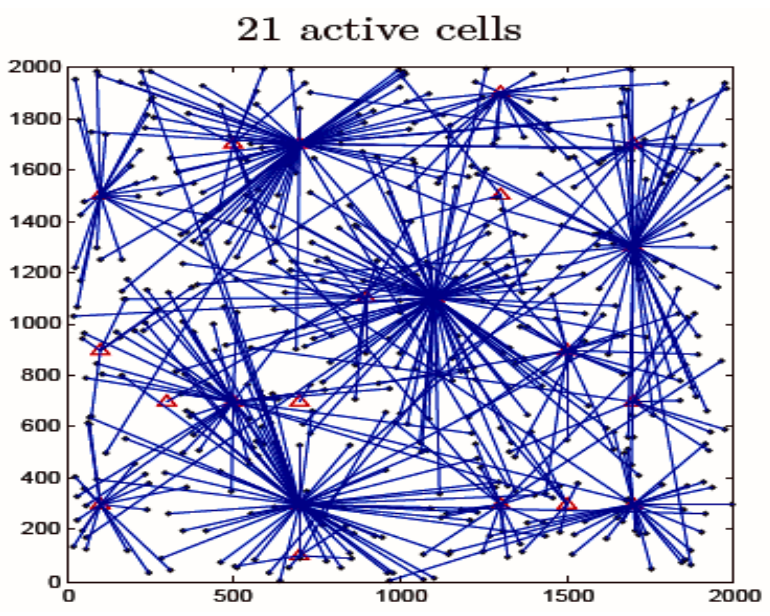

(c) UE based on maximum users- base station distribution

Fig. (3). Effect of UE- base station distribution sequencing criteria, in which case each base station has five UE.

greedy-add method will make the largest base load so that the load of all base stations gets together. When using this greedy-add algorithm to maximize the practice of this base load, it can increase the number of base stations to be closed.

Fig. (2) shows the effect of using three kinds of energysaving sequencing criteria. It is obvious that what sequencing criteria is chosen have a significant impact on the energy savings. Regardless of the amount of each base station UE, the maximum load and maximum center criteria are relatively close to the performance. However, the largest user of energy efficiency criteria for performance is superior to the other two criteria. Regardless of the amount of each base station UE, the performance difference is always at about $5 \%$. The reason for this difference in performance as follows: in the maximum user criteria, the UE according to the number of base stations may serve to determine a next base station to be opened. However, for the maximum load criteria, the selection process is based on the current load of the base station, and does not consider the contribution of UE which is not connected to make a. In the latter case, the selected base station may not be optimal because it dose not make a greater contribution for the UE which is not connected. 
In Fig. (3) each base station has five UE, influence of sequencing criteria on closing procedure and UE-base station distribution method can be seen. Fig. (3a) shows initial UEbase distribution which is before running the CSO. Fig. (3b) and $3 \mathrm{c}$ show the difference of UE- the difference between the base station distribution method. As can be seen from Fig. (3), the concentration of traffic conditions and the opening set of base stations are largely dependent on the base station sequencing criteria.

\section{CONCLUSION}

The CSO method can effectively save energy by close part of the base stations of cellular networks. Most current CSO deployment algorithm closes the base station based on the current load. However, the step of closing base station will be influence by (base sequencing) effects. Therefore, we studied three different sequencing criteria in this article, and compared their impact on the overall energy savings. Simulation results show that the criterion, which is according to the number of UE the base station can serve, is superior to other criteria. In addition, we treat the CSO method as a set covering problems. Based on this representation, we propose a greedy-add algorithm CSO methods. It proved that when the amount of each base station UE is more, this algorithm is superior to Cell-Zooming reference improved algorithms.

\section{ABOUT AUTHOR}

Yi Tian received his M.S. degrees in 2011, from Northwest University of Information Technology. He is now an lecturer in Shangluo University. His research interests include computer system architecture, internet of things.

\section{CONFLICT OF INTEREST}

The authors confirm that this article content has no conflicts of interest.

\section{ACKOWLEDGEMENTS}

This work is supported by a grant from Shaanxi's Department of Education fund (14JK1221).

\section{REFERENCES}

[1] J. Zhu, and L. Xu, "Spectrum-efficiency and energy-efficiency functions of green cellular networks", Journal on Communications, vol. 34, no. 1, pp. 1-7, 2013.

[2] H. Wang, Y. Wang, Y. Huang, and L. Yang, "Pilot contamination reduction in very large MIMO cellular network", Journal on Communications, vol. 35, no. 1, pp. 24-33, 2014

[3] H. Yu, Y. Li, X. Xu, and J. Wang, "Relay cellular network energy efficiency based on stochastic geometry model", Journal of Tsinghua University, vol. 11, pp. 1536-1541, 2013.

[4] Y. Li, "Optimal relays' positions for maximum energy efficiency in cellular networks", Journal of Chongqing University of Posts and Telecommunications(Natural Science Edition), vol. 26, no. 1, pp. 25-30, 2014

[5] Z. Niu, Y. Wu, J. Gong, and Y. Zexi, "Cell zooming for costefficient green cellular networks", IEEE Communications Magazine, vol. 48, no. 11, pp. 74-79, 2010.

[6] F. Alaca, A.B. Sediq, and H. Yanikomeroglu, "A genetic algorithm based cell switch-off scheme for energy saving in dense cell deployments", In: 2012 IEEE Globecom Workshops (GC Wkshps), 2012, pp. 63-68.

[7] S. Zhou, J. Gong, Z. Yang, Z. Niu, and P. Yang, "Green mobile access network with dynamic base station energy saving", In: $A C M$ MobiCom. 2009, vol. 9, no. 262, pp. 10-12.

[8] A. Yildiz, T. Girici, and H. Yanikomeroglu, "A Pricing Based Algorithm for Cell Switching Off in Green Cellular Networks", In: IEEE 77th Vehicular Technology Conference (VTC Spring), 2013, pp. 1-6.

[9] Z. Niu, Y. Wu, J. Gong, and Y. Zexi, "Cell zooming for costefficient green cellular networks", IEEE Communications Magazine, vol. 48, no. 11, pp. 74-79, 2010.

[10] S. Jenjaturong, and C. Intanagonwiwat, "A set cover-based density control algorithm for sensing coverage problems in wireless senso networks", In: IEEE 3rd International Conference on Cognitive Radio Oriented Wireless Networks and Communications, (CrownCom), pp. 1-6.

[11] N. Bansal, R. Krishnaswamy, and B. Saha, "On capacitated set cover problems". In Approximation, Randomization, and Combinatorial Optimization. vol. 6845, Algorithms and Techniques, Ed. Berlin Heidelberg: Springer, 2011, pp. 38-49

[12] Series M, Guidelines for evaluation of radio interface technologies for IMT-Advanced, ITU, Tech. Rep, 2009.

$\begin{array}{lll}\text { Received: September 22, } 2014 & \text { Revised: November 03, } 2014 & \text { Accepted: November 06, 2014 }\end{array}$

(C) Yi Tian; Licensee Bentham Open.

This is an open access article licensed under the terms of the Creative Commons Attribution Non-Commercial License (http://creativecommons.org/licenses/by-nc/3.0/) which permits unrestricted, non-commercial use, distribution and reproduction in any medium, provided the work is properly cited. 